\title{
IDENTIFICATION AND CHARACTERIZATION OF EXCRETORYISECRETORY PRODUCTS OF Giardia duodenalis TROPHOZOITES
}

Thesis: T. Carvalho submitted this dissertation for her Masters in Tropical Diseases at Botucatu Medical School, São Paulo State University, UNESP, Botucatu, São Paulo State, Brazil, 2008.

\section{Advisor: Professor Semíramis Guimarães Ferrraz Viana}

ABSTRACT: One of the major questions concerning Giardia is the understanding of pathophysiological processes associated with small intestine abnormalities. There are evidences that Giardia trophozoites contain and/or release proteolytic enzymes that may be implicated in the host intestinal epithelium. The present investigation was undertaken to examine the protease activity in excretory/secretory (E/S) products of Giardia duodenalis trophozoites of an axenic Brazilian strain (BTU-11) and the reference strain Portland 1 (P1). E/S products from trophozoites of each strain in conditioned medium were tested with sodium dodecyl sulfate polyacrylamide gel electrophoresis (SDS-PAGE) for the protein profiles, and the protease activity was analyzed using substrate-impregnated SDS-PAGE (gelatin and collagen) and hemoglobin assay. The proteases characterization was based on inhibition assays including synthetic inhibitors. Electrophoresis analysis of E/S products revealed a banding pattern composed by few bands ( 4 to 6 bands) in the migration region of 123 to $28 \mathrm{kDa}$. Proteolytic products were detected in the conditioned medium by trophozoites of both assayed strains. In the gels containing copolymerized gelatin and collagen, E/S products promoted substrate degradation and the most evident proteolysis zones were distributed in the migration regions of 77 to $18 \mathrm{kDa}$ and 145 to $18 \mathrm{kDa}$, respectively, in the patterns of gelatinolytic and collagenolytic activities. Degradation of hemoglobin was also observed, and the pattern of hydrolysis was similar in both E/S products assayed. Inhibitor assays showed that the main proteolytic activity in both E/S products is due to cysteine proteases, although the presence of serine proteases was also indicated. Degradation of substrates including 
collagen and hemoglobin could lead us to speculate different functions of Giardia excreted/secreted proteases in vivo, but to confirm this possibility and to elucidate its implication on host-parasite interactions, further experiments applying protocols for the purification of proteases are necessary. Even so, our observations are relevant and hold the perspective for the understanding about protease activity in Giardia trophozoites of axenic strain isolated in an endemic area.

KEY WORDS: Giardia duodenalis, trophozoites, excretory/secretory products, proteases, protein substrates.

\section{CORRESPONDENCE TO:}

THAÍS BATISTA DE CARVALHO, Departamento de Parasitologia, Instituto de Biociências, UNESP, Distrito de Rubião Júnior, s/n, 18618-000, Botucatu, SP, Brazil. Phone: +55 143811 6239. Email: thais_bc@yahoo.com.br. 